\title{
Correction to: Bioplastic from Renewable Biomass: A Facile Solution for a Greener Environment
}

\author{
Gerardo Coppola $^{1} \cdot$ Maria Teresa Gaudio $^{1}$ - Catia Giovanna Lopresto ${ }^{1} \cdot$ Vincenza Calabro $^{1} \cdot$ Stefano Curcio $^{1}$. \\ Sudip Chakraborty ${ }^{1}$ (I)
}

○ King Abdulaziz University and Springer Nature Switzerland AG 2021

\section{Correction to: \\ Earth Systems and Environment (2021) 5:231-251 \\ https://doi.org/10.1007/s41748-021-00208-7}

Unfortunately, conflict of interest statement is missing in the original publication. The conflict of interest is given below:

Conflict of interest: The authors declare that they have no conflict of interest.

We regret the inconvenience caused.

The original article has been updated.

The original article can be found online at https://doi.org/10.1007/ s41748-021-00208-7.

Sudip Chakraborty

sudip.chakraborty@unical.it

1 University of Calabria, Department of DIMES, via Pietro

Bucci, Cubo 42A ${ }^{\circ}, 87036$ Rende, Cosenza, Italy 